\title{
Zur Frage über die Verbindungen des Silbers mit Cadmium.
}

\author{
Von \\ G. J. Petrenko und A. S. Fedorow.
}

Mit 1 Figur im Text und 1 Tafel.

Auf Grund der thermischen und mikroskopischen Untersuchungen haben wir die Existenz der Verbindungen $\mathrm{AgCd}_{4}, \mathrm{AgCd}_{3}, \mathrm{Ag}_{2} \mathrm{Cd}_{3}$ und AgCd angenommen. "Wie bekannt, nehmen auch Brun und QUercigh, die besonders ausführlich die Konzentrationen der Ag-CdLegierungen von $0-20 \% \mathrm{Ag}$ untersuchten, die Existenz der Verbindung $\mathrm{AgCd}_{4}$ an. $^{2}$

Wenn wir auf unser Diagramm ${ }^{3}$ einen Blick werfen, sehen wir, dafs die Verbindungen $\mathrm{AgCd}_{4}$ und $\mathrm{AgCd}_{3}$ Beispiele der Kristallisation der Mischkristalle nach REerschem Typus ${ }^{4}$ darstellen, der eine Ergänzung zu drei von RoozeBoom (1899) abgeleiteten Typen ${ }^{5}$ darbietet. Die Schmelzkurve nach RUER schem Typus hat eine horizontale Tangente im Wendepunkt.

Doch um eine Konzentration, die einem Wendepunkt entspricht, für eine Verbindung anzunehmen, haben wir nur ein Kriterium, nämlich, dafs diese Konzentration dem Gesetze der multiplen Proportionen entsprechen. Und nur im Falle, wenn diese Konzentration im kristallinischen Zustande einen Umwandlungspunkt hat, ${ }^{6}$ kann sie für eine chemische Verbindnng angenommen werden.

Weder $\mathrm{AgCd}_{4}$ noch $\mathrm{AgCd}_{3}$ haben Umwandlungspunkte im kristallinischen Zustande. Für die Existenz der Verbindung $\mathrm{AgCd}_{3}$

I Z. anorg. Chem. 70 (1911), 157-169.

2 Z. anorg. Chem. $68(1910), 198$.

a l. c. S. 161.

4 Zeitschr. phys. Chem. 59 (1907), 4. Beispiele der Kristallisation nach diesem Typus wurden schon von Meerom 'Terwoat im Roozeboomschen Laboratorium (1905) und später von Grobe im Tammansschen Institute (1906) gefunden.

${ }^{5}$ Zeitschr. phys. Chem. $30(1899), 385$.

${ }^{6}$ Grdbe, $Z$. anorg. Chem. 49 (1906), 72. Vgl. auch demnächst erscheinende Untersuchung über die Umwandlungen der Ag-Zn-Legierungen im festen Zustande von G. J. Perrenko. 
spricht die Untersuchung von MaEY. ${ }^{1}$ Aber wenn wir die Unsicherheit der volumetrischen Methode bei der Lösung der Frage über die Type der Verbindungen zwischen zwei Komponenten, die aufser Verbindungen noch isomorphe Mischungen bilden, in Betracht ziehen, so haben wir für die Verbindungen $\mathrm{AgCd}_{4}$ und $\mathrm{AgCd}_{3}$ gleiche Gründe, um ihre Existenz anzunehmen; vielleicht hat $\mathrm{AgCd}_{3}$ mehr Gründe für ihre Existenz, denn ihre Abkühlungskurve hat keinen Intervall, und die Abkühlungskurve, die $\mathrm{AgCd}_{4}$ entspricht, hat einen Intervall von $2{ }^{\circ}$ (Petrenko-Fedorow), obgleich nach den Bestimmungen von BRDNI und QUERcIGH sich diese Konzentration sogar ohne Intervall kristallisiert.

Indem wir vorläufig die Frage über die Existenz der genannten Verbindungen (zu deren Bestätigung wir andere Methoden anwenden könnten, wie z. B. die Bestimmung der elektrischen Leitfähigkeit oder die Untersuchung der ternären Systeme) offen lielsen, hatten wir uns mit der Frage, ob $\mathrm{AgCd}_{4}$ feste Lösungen mit $\mathrm{Cd}$ bildet, beschäftigt, und wenn es der Fall ist, welche Konzentration hat dann der gesättigte Mischkristall.

Um diese Frage $\mathrm{zu}$ entscheiden, waren folgende Versuche angestellt: Reguli mit Gehalt von 18.3-19\% Silber wurden lange Zeit (bis 24 Stunden) bei $320-330^{\circ}$ angelassen; auf diesem Wege wollten wir bestimmen, ob irgendwelche Änderung der Strukturelemente stattfindet.

Auf den Schliffen der Reguli mit 18.3-18.5\% Ag (gewöhnliche Abküblung) sind zwei Strukturelemente zu sehen; das eine erinnert an den Mischkristall $a$, das andere an den Mischkristall $b$.

Das Anlassen der genannten Reguli bei der erwähnten Temperatur hat keine Änderung der relativen Mengen der Strukturelemente zur Folge gehabt; dasselbe gilt auch für den Regulus mit Silbergehalt von $19 \%$. Daraus konnte man schliefsen, dafs, wenn $\mathrm{AgCd}_{4}$ auch feste Lösungen mit $\mathrm{Cd}$ bildet, so übertrifft die Konzentration des gesättigten Mischkristalls $0.3 \tilde{s}_{\%} \mathrm{Cd}$ nicht, weil für die Verbindung $\mathrm{AgCd}_{\mathbb{4}} 19.35 \% \mathrm{Ag}$ berechnet wird.

Nach den genannten Versuchen haben wir die Frage über die Existenz der Verbindung $\mathrm{AgCd}_{4}$ einer sorgfältigeren Untersuchung unterworfen. $\mathrm{Zu}$ diesem $\mathrm{Zwecke}$ haben wir Abschreckungsversuche unternommen. Wenn $\mathrm{AgCd}_{4}$ existiert und bei $530^{\circ}\left(545^{\circ}\right.$ nach Bruni und Quercigh) schmilzt, so wird man offenbar auf den

${ }^{1}$ Zeitschr. phys. Chem. 50 (1904), 207. 
Schliffen der Reguli, die bei $510-520^{\circ}$ abgeschreckt sind, nur polygonale Struktur sehen.

Die Abschreckungsversuche haben zu ganz anderen Resultaten geführt, die, wie wir es gleich sehen werden, die Existenz der Verbindung AgCid nicht bestätigen. Wenn wir bei $510-520^{\circ} \mathrm{ab}-$ schrecken, so sehen wir auf den Schliffen deutlich zwei Strukturelemente; das eine bildet grofse Kristalle von weilser Farbe mit Einschlüssen darin, das andere ist inhomogen und bildet sehr kleine Kristalle. Beim Abschrecken bei verschiedenen Temperaturen im Intervalle von 510 bis $337^{\circ}$ beobachteten wir immer zwei erwähnte Strukturelemente, aber die relativen Mengen derselben ändern sich je nach der Temperatur des Abschreckens: bei böheren Temperaturen ist die Menge des feinkristallinischen Strukturelementes gröfser, als die der grolsen Kristalle, und bei niedrigeren Temperaturen überwiegt die Menge der grofsen

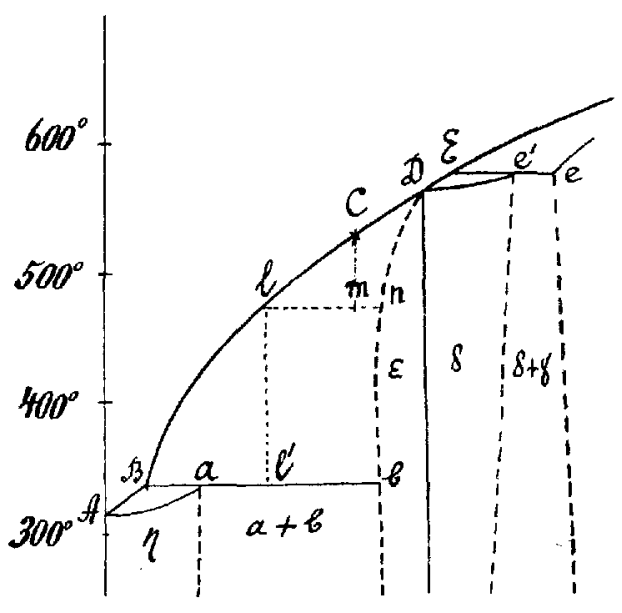
weifsen Kristalle, aber die

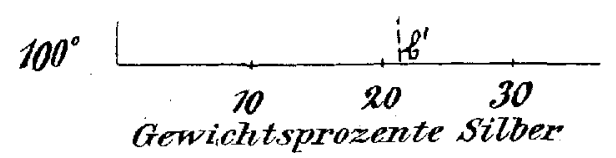

für die Verbindungen und reine chemische Elemente charakteristische polygonale Struktur wird nie beobachtet.

Wenn wir uns zur Figur wenden, die einen Teil unseres Diagrammes, nämlich die Legierungen mit Silbergehalt von $0-36 \%$ darstellt, so so ist es leicht, die beschriebene Struktur der abgeschreckten Reguli zu erklären, mit der Voraussetzung, dals die Verbindung $\mathrm{AgCd}_{4}$ nicht existiert.

Fig. 1, Tafel I ist das Photogramm des Schliffes des Regulus mit $19.5 \% \mathrm{Ag}$, abgeschreckt bei $480^{\circ}$; man sieht weilse kristallinische Massen mit Einschlüssen von grauer Farbe; diese weilsen Kristalle sind von einer heterogenen feinkristallinischen Nasse umgeben. Bei $480^{\circ}$ ist folglich der Mischkristall $n$ im Gleichgewichte 
mit der Schmelze $l$. Beim schnellen Abkühlen bis zur Temperatur des Eiswassers kristallisiert sich die Schmelze $l$ rasch offenbar in das feste Konglomerat $l^{\prime}$, das aus Kristallen $a$ und $b$ besteht.

So müssen wir auf Grund der Abschreckungsversuche schliefsen, dafs die Verbindung $\mathrm{AgCd}_{3}$ nicht existiert. Was aber die Abkühlungskurve für die Konzentration $C$ betrifft, auf der wir einen Haltepunkt von 35 Sekunden, doch mit dem Intervall ron $2^{0}$ angegeben hatten, so war es ein Irrtum, weil wir den Beginn der Kristallisation, dessen Ende wir nicht gefunden hatten, falsch für einen Haltepunkt angenommen haben; bei einem so grolsen Intervalle (etwa $200^{\circ}$ ) ist es fast unmöglich, das Ende der Kristallisation genau zu bestimmen. ${ }^{1}$ In der vorläufigen Mitteilung, die wir Herrn Dr. GUERTLER für sein Handbuch gemacht haben, erwähnten wir sogar diese Verbindung nicht. ${ }^{2}$

Aus der Fig. 2, Tafel I kann man sehen, dafs die Legierung mit Silbergehalt von $19.35 \%$ beim langsamen Abkühlen und Anlassen etwa 24 Stunden, bei $320^{\circ}$ und tiefer keine polygonale Struktur hat. Diese Konzentration kann auch gar nicht einen gesättigten Mischkristall darstellen; der letzte soll die Zusammensetzung des Punktes $b$ (ungefähr 21-21.5\% Ag) haben, weil diese Konzentration nach Anlassen bei $330^{\circ}$ fast homogene Struktur hat. Das Abschrecken der Reguli nach Anlassen bei $320^{\circ}$ hat sehr kleine Änderung der Struktur hervorgerufen, $d . h$. die Linie $b b^{\prime}$ weicht nur wenig nach der Silberseite ab.

Gleichzeitig haben wir Versuche zur Entscheidung der Frage über die Existenz der Verbindung $\mathrm{AgCd}_{3}$ gestellt. Auf den Schliffen der bei 555-560 abgeschreckten Reguli sind Polygone zu sehen (Fig. 3, Tafel I). Es ist möglich, dal's die Verbindung $\mathbf{A g C d}_{3}$ ja existiert, doch um gröfsere Sicherheit darüber zu gewinnen, müssen wohl noch andere Methoden Anwendung finden.

1 TAMmann, $Z$. anorg. Chem. 47 (1905), 301.

* Metallographie 1910, 492.

Charkow (Russland), Phys.-chem. Laboratorium der Universität.

Bei der Redaktion eingegangen am 11. April 1911. 